\title{
Opto-optical gain-clamped L-band Erbium-doped fiber amplifier with C-band control signal
}

\begin{abstract}
We demonstrate an opto-optical gain-clamped L-band erbium-doped fiber amplifier by manipulating the $\mathrm{C}$-band lasing wavelength as the control signal. The L-band gain-clamped value is achieved by tuning the control laser in the C-band wavelength range that propagates in the opposite direction to the L-band signal. Within the wavelength range of $1538 \mathrm{~nm}$ and $1560 \mathrm{~nm}$, the L-band gain decreases linearly with the increment of the C-band lasing wavelength. The L-band gain dynamic range decreases with the increment of the cavity loss. By combining two different levels of cavity loss, the gain dynamic range of $10 \mathrm{~dB}$ from $11 \mathrm{~dB}$ to $21 \mathrm{~dB}$ is achieved with an average noise figure of less than $5.9 \mathrm{~dB}$. The whole gain spectrum of the L-band can be used for multiple-channel amplification because the laser is created outside its signal band.
\end{abstract}

Keyword: Fiber laser; Fiber amplifiers; Optical amplifier; Band gains. 